\title{
On the Achievable Rate Region in the Optimistic Sense for Separate Coding of Two Correlated General Sources*
}

SUMMARY This paper is concerned with coding theorems in the optimistic sense for separate coding of two correlated general sources $\boldsymbol{X}_{1}$ and $\boldsymbol{X}_{2}$. We investigate the achievable rate region $\mathcal{R}_{\mathrm{opt}}\left(\boldsymbol{X}_{1}, \boldsymbol{X}_{2}\right)$ such that the decoding error probability caused by two encoders and one decoder can be arbitrarily small infinitely often under a certain rate constraint. We give an inner and an outer bounds of $\mathcal{R}_{\mathrm{opt}}\left(\boldsymbol{X}_{1}, \boldsymbol{X}_{2}\right)$, where the outer bound is described by using new information-theoretic quantities. We also give two simple sufficient conditions under which the inner bound coincides with the outer bound.

key words: correlated sources, general source, optimistic coding, achievable rate region, information-spectrum methods

\section{Introduction}

In information-spectrum methods that originate from [3] and are described in detail in [4], fixed-length coding of a general source $\boldsymbol{X}=\left\{X^{n}\right\}_{n=1}^{\infty}$ is one of fundamental problems, where, letting $X$ be a finite or countably infinite alphabet, $X^{n}$ is defined as a random variable taking values in $X^{n}$. The class of general sources includes vast classes of sources such as memoryless sources, Markov sources, stationary ergodic sources and stationary sources. Therefore, coding theorems for the class of general sources are valid to such classes of sources.

In coding of a general source we can formulate a new kind of coding problem in the optimistic sense. We are interested in the infimum of the rate $R$ such that there exists a sequence $\left\{\left(\varphi_{n}, \psi_{n}\right)\right\}_{n=1}^{\infty}$ of pairs of an encoder $\varphi_{n}$ and a decoder $\psi_{n}$ satisfying for any $\gamma>0$ both $\frac{1}{n} \log M_{n} \leq R+\gamma$ and $\varepsilon_{n} \leq \gamma$ with a subsequence $n=n_{i}, i \geq 1$, where $M_{n}$ and $\varepsilon_{n}$ denote the number of codewords and the decoding error probability, respectively. This problem was first formulated by Vembu, Verdú and Steinberg [11] and was discussed by Chen and Alajaji [1]. Results related to the optimistic coding problem can be found in [5], [9]. Quite recently, the author defined new information-theoretic quantities and clarified their operational meanings in the optimistic sense [6].

In this paper we discuss the achievable rate region in the optimistic sense for separate coding of a pair $\left(\boldsymbol{X}_{1}, \boldsymbol{X}_{2}\right)=$ $\left\{\left(X_{1}^{n}, X_{2}^{n}\right)\right\}_{n=1}^{\infty}$ of two correlated general sources, where for each $k=1,2 X_{k}^{n} \in X_{k}^{n}$ and $X_{k}$ denotes a finite or countably infinite alphabet. We consider two encoders $\varphi_{n}^{(k)}$ :

Manuscript received February 10, 2012.

Manuscript revised June 25, 2012.

${ }^{\dagger}$ The author is with the Faculty of Systems and Information, University of Tsukuba, Tsukuba-shi, 305-8573 Japan.

${ }^{*}$ A primary version of this paper was presented in [7].

a)E-mail: koga@iit.tsukuba.ac.jp

DOI: 10.1587/transfun.E95.A.2100
$X_{k}^{n} \rightarrow\left\{1,2, \ldots, M_{n}^{(k)}\right\}, k=1,2$, for coding of $X_{1}^{n}$ and $X_{2}^{n}$, respectively, and a decoder $\psi_{n}$ that outputs an estimate $\left(\hat{X}_{1}^{n}, \hat{X}_{2}^{n}\right) \in X_{1}^{n} \times X_{2}^{n}$ of $\left(X_{1}^{n}, X_{2}^{n}\right)$ from two transmitted codewords $I_{n}^{(1)}$ and $I_{n}^{(2)}$ (see Fig. 1). We are interested in the achievable rate region $\mathcal{R}_{\mathrm{opt}}\left(\boldsymbol{X}_{1}, \boldsymbol{X}_{2}\right)$ in the optimistic sense, which is defined as the collection of all the rate pairs $\left(R_{1}, R_{2}\right)$ such that there exists a sequence $\left\{\left(\varphi_{n}^{(1)}, \varphi_{n}^{(2)}, \psi_{n}\right)\right\}_{n=1}^{\infty}$ satisfying $\frac{1}{n} \log M_{n}^{(k)} \leq R_{k}+\gamma(k=1,2)$ and $\varepsilon_{n} \leq \gamma$ for a subsequence $n=n_{i}, i \geq 1$, where $\gamma>0$ is an arbitrarily small constant and $\varepsilon_{n}$ denotes the decoding error probability.

We give an inner and an outer bounds of $\mathcal{R}_{\mathrm{opt}}\left(\boldsymbol{X}_{1}, \boldsymbol{X}_{2}\right)$, where the outer bound can be expressed by using new quantities introduced in [6]. In particular, it is shown that the outer bound is easily treated by characterizing the unachievable region in the strong sense [6]. We also give two simple sufficient conditions under which the outer bound coincides with the inner bound.

The problem of separate coding of correlated sources was first formulated by Slepian and Wolf [10] for the memoryless case. A simple proof using the bin coding is given in Cover [2]. The achievable rate region for general sources $\boldsymbol{X}_{1}$ and $\boldsymbol{X}_{2}$ in the ordinary sense is given in Miyake and Kanaya [8]. The achievable rate region in [8] is extended to the case of $\varepsilon$-error by Han [4]. However, to the author's knowledge, no coding theorem in the optimistic sense is obtained so far. Our approach using the inner and the outer bounds clarifies a difference between coding in the ordinary sense and in the optimistic sense.

\section{Problem Setup}

In this section we consider separate coding of two correlated sources [10]. The block diagram is given in Fig. 1. Let $\left(\boldsymbol{X}_{1}, \boldsymbol{X}_{2}\right)=\left\{\left(X_{1}^{n}, X_{2}^{n}\right)\right\}_{n=1}^{\infty}$ be two correlated general sources. For each $n \geq 1 X_{1}^{n}$ and $X_{2}^{n}$ take values in $X_{1}^{n}$ and $X_{2}^{n}$, respectively, where $X_{1}$ and $X_{2}$ denote finite or countably infinite alphabets. Denote by $P_{X_{1}^{n} X_{2}^{n}}$ and $P_{X_{k}^{n} \mid X_{l}^{n}}$ the joint probabil-

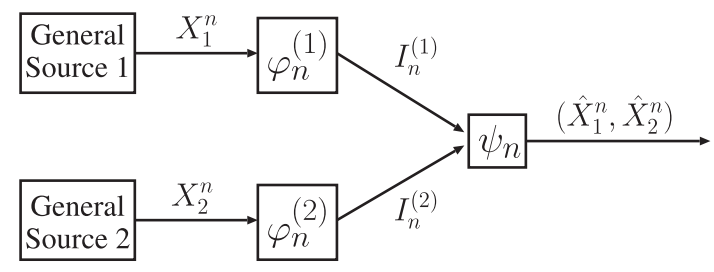

Fig. 1 Separate coding of two correlated general sources. 
ity distribution of $\left(X_{1}^{n}, X_{2}^{n}\right)$ and the conditional probability distribution of $X_{k}^{n}$ given $X_{l}^{n}$ for $(k, l)=(1,2)$ and $(2,1)$. For each $k=1,2$ we define an encoder $k$ as a deterministic mapping $\varphi_{n}^{(k)}: X_{k}^{n} \rightarrow \mathcal{M}_{n}^{(k)} \stackrel{\text { def }}{=}\left\{1,2, \ldots, M_{n}^{(k)}\right\}$. In addition, we define a decoder as a deterministic mapping $\psi_{n}: \mathcal{M}_{n}^{(1)} \times \mathcal{M}_{n}^{(2)} \rightarrow \mathcal{X}_{1}^{n} \times \mathcal{X}_{2}^{n}$. The decoding error probability caused by the triplet $\left(\varphi_{n}^{(1)}, \varphi_{n}^{(2)}, \psi_{n}\right)$ is defined by

$$
\varepsilon_{n}=\operatorname{Pr}\left\{\psi_{n}\left(\varphi_{n}^{(1)}\left(X_{1}^{n}\right), \varphi_{n}^{(2)}\left(X_{2}^{n}\right)\right) \neq\left(X_{1}^{n}, X_{2}^{n}\right)\right\},
$$

where throughout this paper $\operatorname{Pr}\{\cdot\}$ denotes the probability with respect to the joint probability $P_{X_{1}^{n} X_{2}^{n}}$. Hereinafter, we call a sequence $\left\{\left(\varphi_{n}^{(1)}, \varphi_{n}^{(2)}, \psi_{n}\right)\right\}_{n=1}^{\infty}$ of the triplets of two encoders $\varphi_{n}^{(1)}$ and $\varphi_{n}^{(2)}$ and a decoder $\psi_{n}$ a code for short.

Miyake and Kanaya [8] obtained the achievable rate region under the requirement of $\varepsilon_{n} \rightarrow 0$ as $n \rightarrow \infty$.

Definition 1: A rate pair $\left(R_{1}, R_{2}\right)$ is called achievable in the ordinary sense if there exists a code $\left\{\left(\varphi_{n}^{(1)}, \varphi_{n}^{(2)}, \psi_{n}\right)\right\}_{n=1}^{\infty}$ satisfying

$$
\limsup _{n \rightarrow \infty} \frac{1}{n} \log M_{n}^{(k)} \leq R_{k}, \quad k=1,2
$$

and

$$
\lim _{n \rightarrow \infty} \varepsilon_{n}=0
$$

where $\log (\cdot)=\log _{2}(\cdot)$ throughout this paper. Define the achievable rate region in the ordinary sense by

$$
\begin{aligned}
& \mathcal{R}\left(X_{1}, X_{2}\right)=\operatorname{Cl}\left(\left\{\left(R_{1}, R_{2}\right):\right.\right. \\
& \left.\left.\quad\left(R_{1}, R_{2}\right) \text { is achievable in the ordinary sense }\right\}\right),
\end{aligned}
$$

where $\mathrm{Cl}(\cdot)$ denotes the closure of the set.

Theorem 1 ([8]): It holds that

$$
\mathcal{R}\left(X_{1}, X_{2}\right)=\mathcal{S}\left(X_{1}, X_{2}\right),
$$

where

$$
\begin{aligned}
& \mathcal{S}\left(\boldsymbol{X}_{1}, \boldsymbol{X}_{2}\right)=\left\{\left(R_{1}, R_{2}\right): R_{1} \geq \bar{H}\left(\boldsymbol{X}_{1} \mid \boldsymbol{X}_{2}\right),\right. \\
& \left.\quad R_{2} \geq \bar{H}\left(\boldsymbol{X}_{2} \mid \boldsymbol{X}_{1}\right) \text { and } R_{1}+R_{2} \geq \bar{H}\left(\boldsymbol{X}_{1}, \boldsymbol{X}_{2}\right)\right\}
\end{aligned}
$$

and

$$
\begin{aligned}
& \bar{H}\left(\boldsymbol{X}_{1}, \boldsymbol{X}_{2}\right)=\inf \{\alpha: \\
& \left.\liminf _{n \rightarrow \infty} \operatorname{Pr}\left\{\frac{1}{n} \log \frac{1}{P_{X_{1}{ }^{n} X_{2}{ }^{n}}\left(X_{1}^{n}, X_{2}^{n}\right)} \leq \alpha\right\}=1\right\}, \\
& \bar{H}\left(\boldsymbol{X}_{k} \mid \boldsymbol{X}_{l}\right)=\inf \{\alpha: \\
& \left.\liminf _{n \rightarrow \infty} \operatorname{Pr}\left\{\frac{1}{n} \log \frac{1}{P_{X_{k}{ }^{n} \mid X_{l}}\left(X_{k}^{n} \mid X_{l}^{n}\right)} \leq \alpha\right\}=1\right\}
\end{aligned}
$$

for $(k, l)=(1,2)$ and $(2,1)$.

It is important to notice that (1) and (2) require that for any $\gamma>0 \frac{1}{n} \log M_{n}^{(k)} \leq R_{k}+\gamma(k=1,2)$ and $\varepsilon_{n} \leq \gamma$ for all sufficiently large $n$.

In this paper, we are interested in the achievable rate region in the optimistic sense defined as follows:

Definition 2: A rate pair $\left(R_{1}, R_{2}\right)$ is called achievable in the optimistic sense if there exists a code $\left\{\left(\varphi_{n}^{(1)}, \varphi_{n}^{(2)}, \psi_{n}\right)\right\}_{n=1}^{\infty}$ such that for any $\gamma>0$ there exists a subsequence $\left\{n_{i}\right\}_{i=1}^{\infty}$ satisfying

$$
\frac{1}{n} \log M_{n}^{(k)} \leq R_{k}+\gamma, \quad k=1,2
$$

and

$$
\varepsilon_{n} \leq \gamma
$$

for all $n=n_{i}, i \geq 1$. Define the achievable rate region in the optimistic sense by

$$
\begin{aligned}
& \mathcal{R}_{\text {opt }}\left(X_{1}, X_{2}\right)=\operatorname{Cl}\left(\left\{\left(R_{1}, R_{2}\right):\right.\right. \\
& \left.\left.\quad\left(R_{1}, R_{2}\right) \text { is achievable in the optimistic sense }\right\}\right) .
\end{aligned}
$$

Note that in Definition 2 (3) and (4) require that for any $\gamma>0 \frac{1}{n} \log M_{n}^{(k)} \leq R_{k}+\gamma(k=1,2)$ and $\varepsilon_{n} \leq \gamma$ simultaneously for infinitely many $n$. Hence, it is obvious that that $\mathcal{R}\left(\boldsymbol{X}_{1}, \boldsymbol{X}_{2}\right) \subset \mathcal{R}_{\mathrm{opt}}\left(\boldsymbol{X}_{1}, \boldsymbol{X}_{2}\right)$. While the formula of the infimum achievable rate in the optimistic sense for fixed-tofixed length coding of a general source $\boldsymbol{X}$ is given in [1], no result is known on the achievable rate region $\mathcal{R}_{\text {opt }}\left(\boldsymbol{X}_{1}, \boldsymbol{X}_{2}\right)$ in the optimistic sense.

We also consider the following region.

Definition 3: A rate pair $\left(R_{1}, R_{2}\right)$ is called unachievable in the strong sense if for any code $\left\{\left(\varphi_{n}^{(1)}, \varphi_{n}^{(2)}, \psi_{n}\right)\right\}_{n=1}^{\infty}$ satisfying (1) it holds that

$$
\liminf _{n \rightarrow \infty} \varepsilon_{n}>0 .
$$

Define the unachievable rate region in the strong sense by

$$
\begin{aligned}
& \mathcal{U}\left(X_{1}, X_{2}\right)=\left\{\left(R_{1}, R_{2}\right):\right. \\
& \left.\quad\left(R_{1}, R_{2}\right) \text { is unachievable in the strong sense }\right\} .
\end{aligned}
$$

Notice that (5) means that $\varepsilon_{n}$ is positive for all sufficiently large $n$. This $\mathcal{U}\left(\boldsymbol{X}_{1}, \boldsymbol{X}_{2}\right)$ is closely related to $\mathcal{R}_{\text {opt }}\left(\boldsymbol{X}_{1}, \boldsymbol{X}_{2}\right)$ and facilitates characterization of $\mathcal{R}_{\mathrm{opt}}\left(\boldsymbol{X}_{1}, \boldsymbol{X}_{2}\right)$.

The following proposition is easily obtained from the definitions of $\mathcal{R}_{\mathrm{opt}}\left(\boldsymbol{X}_{1}, \boldsymbol{X}_{2}\right)$ and $\mathcal{U}\left(\boldsymbol{X}_{1}, \boldsymbol{X}_{2}\right)$.

Proposition 1: Define $\mathcal{U}^{\dagger}\left(\boldsymbol{X}_{1}, \boldsymbol{X}_{2}\right)=\mathrm{Cl}\left(\mathcal{U}^{c}\left(\boldsymbol{X}_{1}, \boldsymbol{X}_{2}\right)\right)$, where the superscript $c$ denotes the complement. Then, it holds that $\mathcal{U}^{\dagger}\left(\boldsymbol{X}_{1}, \boldsymbol{X}_{2}\right) \subset \mathcal{R}_{\text {opt }}\left(\boldsymbol{X}_{1}, \boldsymbol{X}_{2}\right)$.

Proof: If $\left(R_{1}, R_{2}\right) \in \mathcal{U}^{c}\left(X_{1}, X_{2}\right)$, there exists a code $\left\{\left(\varphi_{n}^{(1)}, \varphi_{n}^{(2)}, \psi_{n}\right)\right\}_{n=1}^{\infty}$ satisfying (1) and $\liminf _{n \rightarrow \infty} \varepsilon_{n}=0$. Such a code $\left\{\left(\varphi_{n}^{(1)}, \varphi_{n}^{(2)}, \psi_{n}\right)\right\}_{n=1}^{\infty}$ satisfies, letting $\gamma>0$ be an arbitrary constant, $\frac{1}{n} \log M_{n}^{(k)} \leq R_{k}+\gamma, k=1,2$, for all sufficiently large $n$ and $\varepsilon_{n} \leq \gamma$ infinitely often. This means $\mathcal{U}^{c}\left(\boldsymbol{X}_{1}, \boldsymbol{X}_{2}\right) \subset \mathcal{R}_{\text {opt }}\left(\boldsymbol{X}_{1}, \boldsymbol{X}_{2}\right)$ because $\left(R_{1}, R_{2}\right) \in \mathcal{U}^{c}\left(\boldsymbol{X}_{1}, \boldsymbol{X}_{2}\right)$ is arbitrary. Since $\mathcal{R}_{\text {opt }}\left(\boldsymbol{X}_{1}, \boldsymbol{X}_{2}\right)$ is defined as the closure, the claim of this proposition follows. 
Remark: In [4], [8] the achievable rate region $\mathcal{R}\left(\boldsymbol{X}_{1}, \boldsymbol{X}_{2}\right)$ is defined without taking the closure. In this paper, however, $\mathcal{R}\left(\boldsymbol{X}_{1}, \boldsymbol{X}_{2}\right)$ and $\mathcal{R}_{\text {opt }}\left(\boldsymbol{X}_{1}, \boldsymbol{X}_{2}\right)$ are defined as the closures so that we can ensure technical soundness especially of Proposition 1 and discussions in Sect. 4.1.

\section{Main Results}

Before giving inner and outer bounds of $\mathcal{R}_{\mathrm{opt}}\left(\boldsymbol{X}_{1}, \boldsymbol{X}_{2}\right)$ and $\mathcal{U}\left(\boldsymbol{X}_{1}, \boldsymbol{X}_{2}\right)$, we define the three events for $R \geq 0$ as follows:

$$
\begin{aligned}
& E_{n}^{(1)}(R)=\left\{\left(x_{1}^{n}, x_{2}^{n}\right) \in \mathcal{X}_{1}^{n} \times \mathcal{X}_{2}^{n}: \frac{1}{n} \log \frac{1}{P_{X_{1}^{n} \mid X_{2}^{n}}\left(x_{1}^{n} \mid x_{2}^{n}\right)} \geq R\right\}, \\
& E_{n}^{(2)}(R)=\left\{\left(x_{1}^{n}, x_{2}^{n}\right) \in \mathcal{X}_{1}^{n} \times \mathcal{X}_{2}^{n}: \frac{1}{n} \log \frac{1}{\left.P_{X_{2}^{n} \mid X_{1}^{n}\left(x_{2}^{n} \mid x_{1}^{n}\right)} \geq R\right\},}\right. \\
& E_{n}^{(3)}(R)=\left\{\left(x_{1}^{n}, x_{2}^{n}\right) \in \mathcal{X}_{1}^{n} \times \mathcal{X}_{2}^{n}: \frac{1}{n} \log \frac{1}{P_{X_{1}^{n} X_{2}^{n}}\left(x_{1}^{n}, x_{2}^{n}\right)} \geq R\right\} .
\end{aligned}
$$

Furthermore, define

$$
\begin{gathered}
\mathcal{A}\left(\boldsymbol{X}_{1}, \boldsymbol{X}_{2}\right)=\left\{\left(R_{1}, R_{2}\right): \text { for any } \gamma>0\right. \\
\liminf _{n \rightarrow \infty} \operatorname{Pr}\left\{E_{n}^{(1)}\left(R_{1}+\gamma\right) \cup E_{n}^{(2)}\left(R_{2}+\gamma\right)\right. \\
\left.\left.\cup E_{n}^{(3)}\left(R_{1}+R_{2}+\gamma\right)\right\}=0 \text { holds }\right\}
\end{gathered}
$$

and

$$
\begin{gathered}
\mathcal{B}\left(\boldsymbol{X}_{1}, \boldsymbol{X}_{2}\right)=\left\{\left(R_{1}, R_{2}\right): \text { for any } \gamma>0\right. \text { all of } \\
\liminf _{n \rightarrow \infty} \operatorname{Pr}\left\{E_{n}^{(1)}\left(R_{1}+\gamma\right)\right\}=0, \\
\liminf _{n \rightarrow \infty} \operatorname{Pr}\left\{E_{n}^{(2)}\left(R_{2}+\gamma\right)\right\}=0, \\
\left.\liminf _{n \rightarrow \infty} \operatorname{Pr}\left\{E_{n}^{(3)}\left(R_{1}+R_{2}+\gamma\right)\right\}=0 \text { hold }\right\} .
\end{gathered}
$$

In fact, $\mathcal{B}\left(\boldsymbol{X}_{1}, \boldsymbol{X}_{2}\right)$ can be written in the following form.

Proposition 2: $\mathcal{B}\left(X_{1}, X_{2}\right)=\mathcal{S}^{*}\left(X_{1}, X_{2}\right)$, where

$$
\begin{aligned}
& \mathcal{S}^{*}\left(\boldsymbol{X}_{1}, \boldsymbol{X}_{2}\right)=\left\{\left(R_{1}, R_{2}\right): R_{1} \geq \bar{H}^{*}\left(\boldsymbol{X}_{1} \mid \boldsymbol{X}_{2}\right),\right. \\
& \left.\quad R_{2} \geq \bar{H}^{*}\left(\boldsymbol{X}_{2} \mid \boldsymbol{X}_{1}\right) \text { and } R_{1}+R_{2} \geq \bar{H}^{*}\left(\boldsymbol{X}_{1}, \boldsymbol{X}_{2}\right)\right\}
\end{aligned}
$$

and

$$
\begin{aligned}
& \bar{H}^{*}\left(\boldsymbol{X}_{1}, \boldsymbol{X}_{2}\right)=\inf \{\alpha: \\
& \limsup _{n \rightarrow \infty} \operatorname{Pr}\left\{\frac{1}{n} \log \frac{1}{\left.\left.P_{X_{1}^{n} X_{2}^{n}\left(X_{1}^{n}, X_{2}^{n}\right)} \leq \alpha\right\}=1\right\},}\right. \\
& \bar{H}^{*}\left(\boldsymbol{X}_{k} \mid \boldsymbol{X}_{l}\right)=\inf \{\alpha: \\
& \left.\limsup _{n \rightarrow \infty} \operatorname{Pr}\left\{\frac{1}{n} \log \frac{1}{P_{X_{k}^{n} \mid X_{l}^{n}}\left(X_{k}^{n} \mid X_{l}^{n}\right)} \leq \alpha\right\}=1\right\}
\end{aligned}
$$

for $(k, l)=(1,2)$ and $(2,1)$.

Proof: We first prove $\mathcal{B}\left(\boldsymbol{X}_{1}, \boldsymbol{X}_{2}\right) \subset \mathcal{S}^{*}\left(\boldsymbol{X}_{1}, \boldsymbol{X}_{2}\right)$. Let $\left(R_{1}, R_{2}\right)$ be an arbitrary element of $\mathcal{B}\left(\boldsymbol{X}_{1}, \boldsymbol{X}_{2}\right)$. Then, letting $\gamma>0$ be an arbitrary constant, we have

$$
\liminf _{n \rightarrow \infty} \operatorname{Pr}\left\{E_{n}^{(1)}\left(R_{1}+\gamma\right)\right\}
$$

$$
=\liminf _{n \rightarrow \infty} \operatorname{Pr}\left\{\frac{1}{n} \log \frac{1}{P_{X_{1}^{n} \mid X_{2}^{n}}\left(X_{1}^{n} \mid X_{2}^{n}\right)} \geq R_{1}+\gamma\right\}=0,
$$

which implies that

$$
\limsup _{n \rightarrow \infty} \operatorname{Pr}\left\{\frac{1}{n} \log \frac{1}{P_{X_{1}^{n} \mid X_{2}^{n}}\left(X_{1}^{n} \mid X_{2}^{n}\right)} \leq R_{1}+\gamma\right\}=1
$$

for any $\gamma>0$. Note that we can use $\leq$ instead of $<$ in (8). Hence, it holds that $\bar{H}^{*}\left(\boldsymbol{X}_{1} \mid \boldsymbol{X}_{2}\right) \leq R_{1}$ owing to the definition of $\bar{H}^{*}\left(\boldsymbol{X}_{1} \mid \boldsymbol{X}_{2}\right)$. Similarly, we can obtain $\bar{H}^{*}\left(X_{2} \mid X_{1}\right) \leq R_{2}$ and $\bar{H}^{*}\left(X_{1} X_{2}\right) \leq R_{1}+R_{2}$. Thus, we obtain $\left(R_{1}, R_{2}\right) \in \mathcal{S}^{*}\left(\boldsymbol{X}_{1}, \boldsymbol{X}_{2}\right)$. Since $\left(R_{1}, R_{2}\right) \in \mathcal{B}\left(\boldsymbol{X}_{1}, \boldsymbol{X}_{2}\right)$ is arbitrary, $\mathcal{B}\left(\boldsymbol{X}_{1}, \boldsymbol{X}_{2}\right) \subset \mathcal{S}^{*}\left(\boldsymbol{X}_{1}, \boldsymbol{X}_{2}\right)$ is established.

Next, we prove $\mathcal{S}^{*}\left(\boldsymbol{X}_{1}, \boldsymbol{X}_{2}\right) \subset \mathcal{B}\left(\boldsymbol{X}_{1}, \boldsymbol{X}_{2}\right)$. Fix $\left(R_{1}, R_{2}\right) \in$ $\mathcal{S}^{*}\left(X_{1}, X_{2}\right)$ arbitrarily. Since $R_{1} \geq \bar{H}^{*}\left(\boldsymbol{X}_{1} \mid \boldsymbol{X}_{2}\right)$ is guaranteed, for any $\gamma>0$ it follows that

$$
\begin{aligned}
& \operatorname{Pr}\left\{E_{n}^{(1)}\left(R_{1}+\gamma\right)\right\}=\operatorname{Pr}\left\{\frac{1}{n} \log \frac{1}{P_{X_{1}^{n} \mid X_{2}^{n}}\left(X_{1}^{n} \mid X_{2}^{n}\right)} \geq R_{1}+\gamma\right\} \\
& \leq \operatorname{Pr}\left\{\frac{1}{n} \log \frac{1}{P_{X_{1}^{n} \mid X_{2}^{n}}\left(X_{1}^{n} \mid X_{2}^{n}\right)}>\bar{H}^{*}\left(X_{1} \mid X_{2}\right)+\frac{\gamma}{2}\right\} \\
& =1-\operatorname{Pr}\left\{\frac{1}{n} \log \frac{1}{P_{X_{1}^{n} \mid X_{2}^{n}}\left(X_{1}^{n} \mid X_{2}^{n}\right)} \leq \bar{H}^{*}\left(X_{1} \mid X_{2}\right)+\frac{\gamma}{2}\right\} .
\end{aligned}
$$

By taking the limit inferior of both sides, we have

$$
\begin{aligned}
& \liminf _{n \rightarrow \infty} \operatorname{Pr}\left\{E_{n}^{(1)}\left(R_{1}+\gamma\right)\right\} \\
& \leq 1-\limsup _{n \rightarrow \infty} \operatorname{Pr}\left\{\frac{1}{n} \log \frac{1}{P_{X_{1}^{n} \mid X_{2}^{n}}\left(X_{1}^{n} \mid X_{2}^{n}\right)} \leq \bar{H}^{*}\left(X_{1} \mid X_{2}\right)+\frac{\gamma}{2}\right\} \\
& =0
\end{aligned}
$$

due to the definition of $\bar{H}^{*}\left(\boldsymbol{X}_{1} \mid \boldsymbol{X}_{2}\right)$. Since $\operatorname{Pr}\left\{E_{n}^{(1)}\left(R_{1}+\gamma\right)\right\} \geq$ 0 for all $n \geq 1$, this establishes $\liminf _{n \rightarrow \infty} \operatorname{Pr}\left\{E_{n}^{(1)}\left(R_{1}+\gamma\right)\right\}=$ 0 . Similarly, we can prove $\liminf _{n \rightarrow \infty} \operatorname{Pr}\left\{E_{n}^{(2)}\left(R_{2}+\gamma\right)\right\}=0$ and $\liminf _{n \rightarrow \infty} \operatorname{Pr}\left\{E_{n}^{(3)}\left(R_{1}+R_{2}+\gamma\right)\right\}=0$. Thus, we obtain $\left(R_{1}, R_{2}\right) \in \mathcal{B}\left(\boldsymbol{X}_{1}, \boldsymbol{X}_{2}\right)$. Since $\left(R_{1}, R_{2}\right) \in \mathcal{S}^{*}\left(\boldsymbol{X}_{1}, \boldsymbol{X}_{2}\right)$ is arbitrary, $\mathcal{S}^{*}\left(\boldsymbol{X}_{1}, \boldsymbol{X}_{2}\right) \subset \mathcal{B}\left(\boldsymbol{X}_{1}, \boldsymbol{X}_{2}\right)$ follows.

Proposition 3: $\mathcal{A}\left(\boldsymbol{X}_{1}, \boldsymbol{X}_{2}\right) \subset \mathcal{B}\left(\boldsymbol{X}_{1}, \boldsymbol{X}_{2}\right)$.

Proof: For any $\left(R_{1}, R_{2}\right) \in \mathcal{A}\left(X_{1}, X_{2}\right)$ it holds that

$$
\begin{aligned}
& \liminf _{n \rightarrow \infty} \operatorname{Pr}\left\{E_{n}^{(1)}\left(R_{1}+\gamma\right)\right\}=\liminf _{n \rightarrow \infty} \operatorname{Pr}\left\{E_{n}^{(2)}\left(R_{2}+\gamma\right)\right\} \\
& \quad=\liminf _{n \rightarrow \infty} \operatorname{Pr}\left\{E_{n}^{(3)}\left(R_{1}+R_{2}+\gamma\right)\right\}=0
\end{aligned}
$$

because all of $E_{n}^{(1)}\left(R_{1}+\gamma\right), E_{n}^{(2)}\left(R_{2}+\gamma\right)$ and $E_{n}^{(3)}\left(R_{1}+R_{2}+\gamma\right)$ are subsets of $E_{n}^{(1)}\left(R_{1}+\gamma\right) \cup E_{n}^{(2)}\left(R_{2}+\gamma\right) \cup E_{n}^{(3)}\left(R_{1}+R_{2}+\gamma\right)$. Thus, the claim of this proposition follows immediately.

Now, we are ready to state the main results.

Theorem 2: For any two correlated general sources $\boldsymbol{X}_{1}$ and $\boldsymbol{X}_{2}$ we have

$$
\mathcal{A}\left(\boldsymbol{X}_{1}, \boldsymbol{X}_{2}\right) \subset \mathcal{U}^{\dagger}\left(\boldsymbol{X}_{1}, \boldsymbol{X}_{2}\right) \subset \mathcal{R}_{\mathrm{opt}}\left(\boldsymbol{X}_{1}, \boldsymbol{X}_{2}\right) \subset \mathcal{B}\left(\boldsymbol{X}_{1}, \boldsymbol{X}_{2}\right) .
$$
orem 2 .

We use the following two lemmas in the proof of The- 
Lemma 1 ([4]): Let $M_{n}^{(1)}$ and $M_{n}^{(2)}$ be positive integers arbitrarily given. Then, there exists a code $\left\{\left(\varphi_{n}^{(1)}, \varphi_{n}^{(2)}, \psi_{n}\right)\right\}_{n=1}^{\infty}$ satisfying

$$
\begin{aligned}
& \varepsilon_{n} \leq \operatorname{Pr}\left\{\frac{1}{n} \log \frac{1}{P_{X_{1}^{n} \mid X_{2}^{n}}\left(X_{1}^{n} \mid X_{2}^{n}\right)} \geq \frac{1}{n} \log M_{n}^{(1)}-\gamma\right. \\
& \text { or } \frac{1}{n} \log \frac{1}{P_{X_{2}^{n} \mid X_{1}^{n}}\left(X_{2}^{n} \mid X_{1}^{n}\right)} \geq \frac{1}{n} \log M_{n}^{(2)}-\gamma \\
& \text { or } \left.\frac{1}{n} \log \frac{1}{P_{X_{1}^{n} X_{2}^{n}}\left(X_{1}^{n} X_{2}^{n}\right)} \geq \frac{1}{n} \log M_{n}^{(1)} M_{n}^{(2)}-\gamma\right\} \\
& +3 \cdot 2^{-n \gamma}
\end{aligned}
$$

for any $\gamma>0$ and $n \geq 1$.

Lemma 2 ([4]): Any code $\left\{\left(\varphi_{n}^{(1)}, \varphi_{n}^{(2)}, \psi_{n}\right)\right\}_{n=1}^{\infty}$ satisfies

$$
\begin{gathered}
\varepsilon_{n} \geq \operatorname{Pr}\left\{\frac{1}{n} \log \frac{1}{P_{X_{1}^{n} \mid X_{2}^{n}}\left(X_{1}^{n} \mid X_{2}^{n}\right)} \geq \frac{1}{n} \log M_{n}^{(1)}+\gamma\right. \\
\quad \text { or } \frac{1}{n} \log \frac{1}{P_{X_{2}^{n} \mid X_{1}^{n}}\left(X_{2}^{n} \mid X_{1}^{n}\right)} \geq \frac{1}{n} \log M_{n}^{(2)}+\gamma \\
\text { or } \left.\frac{1}{n} \log \frac{1}{P_{X_{1}^{n} X_{2}^{n}}\left(X_{1}^{n} X_{2}^{n}\right)} \geq \frac{1}{n} \log M_{n}^{(1)} M_{n}^{(2)}+\gamma\right\} \\
-3 \cdot 2^{-n \gamma}
\end{gathered}
$$

for all $n \geq 1$ and $\gamma>0$.

Proof of Theorem 2: $\quad$ Since Proposition 1 guarantees $\mathcal{U}^{\dagger}\left(\boldsymbol{X}_{1}, \boldsymbol{X}_{2}\right) \subset \mathcal{R}_{\text {opt }}\left(\boldsymbol{X}_{1}, \boldsymbol{X}_{2}\right)$, we have only to prove $\mathcal{A}\left(\boldsymbol{X}_{1}, \boldsymbol{X}_{2}\right) \subset \mathcal{U}^{\dagger}\left(\boldsymbol{X}_{1}, \boldsymbol{X}_{2}\right)$ and $\mathcal{R}_{\mathrm{opt}}\left(\boldsymbol{X}_{1}, \boldsymbol{X}_{2}\right) \subset \mathcal{B}\left(\boldsymbol{X}_{1}, \boldsymbol{X}_{2}\right)$.

First, we prove $\mathcal{A}\left(\boldsymbol{X}_{1}, \boldsymbol{X}_{2}\right) \subset \mathcal{U}^{\dagger}\left(\boldsymbol{X}_{1}, \boldsymbol{X}_{2}\right)$. We use the idea in [4, Theorem 7.4.1]. Fix $\gamma>0$ arbitrarily. We first show that $\left(R_{1}, R_{2}\right) \in \mathcal{A}\left(X_{1}, X_{2}\right)$ implies $\left(R_{1}+2 \gamma, R_{2}+2 \gamma\right) \in$ $\mathcal{U}^{c}\left(\boldsymbol{X}_{1}, \boldsymbol{X}_{2}\right)$. Set $M_{n}^{(1)}=2^{n\left(R_{1}+2 \gamma\right)}$ and $M_{n}^{(2)}=2^{n\left(R_{2}+2 \gamma\right)}$. Clearly, these $M_{n}^{(1)}$ and $M_{n}^{(2)}$ satisfy

$$
\limsup _{n \rightarrow \infty} \frac{1}{n} \log M_{n}^{(k)} \leq R_{k}+2 \gamma, \quad k=1,2 .
$$

Since Lemma 1 guarantees the existence of a code $\left\{\left(\varphi_{n}^{(1)}, \varphi_{n}^{(2)}, \psi_{n}\right)\right\}_{n=1}^{\infty}$ satisfying (9). we have

$$
\begin{gathered}
\varepsilon_{n} \leq \operatorname{Pr}\left\{\frac{1}{n} \log \frac{1}{P_{X_{1}^{n} \mid X_{2}^{n}}\left(X_{1}^{n} \mid X_{2}^{n}\right)} \geq R_{1}+\gamma\right. \\
\quad \text { or } \frac{1}{n} \log \frac{1}{P_{X_{2}^{n} \mid X_{1}^{n}}\left(X_{2}^{n} \mid X_{1}^{n}\right)} \geq R_{2}+\gamma \\
\left.\quad \text { or } \frac{1}{n} \log \frac{1}{P_{X_{1}^{n} X_{2}^{n}}\left(X_{1}^{n} X_{2}^{n}\right)} \geq R_{1}+R_{2}+3 \gamma\right\} \\
+3 \cdot 2^{-n \gamma} \\
\leq \operatorname{Pr}\left\{E_{n}^{(1)}\left(R_{1}+\gamma\right) \cup E_{n}^{(2)}\left(R_{2}+\gamma\right)\right. \\
\left.\cup E_{n}^{(3)}\left(R_{1}+R_{2}+\gamma\right)\right\}+3 \cdot 2^{-n \gamma} .
\end{gathered}
$$

Since $\left(R_{1}, R_{2}\right) \in \mathcal{A}\left(\boldsymbol{X}_{1}, \boldsymbol{X}_{2}\right)$ by assumption, by taking the limit inferior of both sides we have

$$
\liminf _{n \rightarrow \infty} \varepsilon_{n}=0,
$$

which, together with (11), implies that $\left(R_{1}+2 \gamma, R_{2}+2 \gamma\right) \in$
$\mathcal{U}^{c}\left(\boldsymbol{X}_{1}, \boldsymbol{X}_{2}\right)$. Since $\gamma>0$ is arbitrary, by letting $\gamma \downarrow 0$ we can conclude that $\left(R_{1}, R_{2}\right) \in \mathcal{U}^{\dagger}\left(\boldsymbol{X}_{1}, \boldsymbol{X}_{2}\right)$. This completes the proof of $\mathcal{A}\left(\boldsymbol{X}_{1}, \boldsymbol{X}_{2}\right) \subset \mathcal{U}^{\dagger}\left(\boldsymbol{X}_{1}, \boldsymbol{X}_{2}\right)$.

Next, we prove $\mathcal{R}_{\text {opt }}\left(\boldsymbol{X}_{1}, \boldsymbol{X}_{2}\right) \subset \mathcal{B}\left(\boldsymbol{X}_{1}, \boldsymbol{X}_{2}\right)$. In view of Proposition 2, it suffices to prove that any $\left(R_{1}, R_{2}\right) \in$ $\mathcal{R}_{\text {opt }}\left(\boldsymbol{X}_{1}, \boldsymbol{X}_{2}\right)$ belongs to $\mathcal{S}^{*}\left(\boldsymbol{X}_{1}, \boldsymbol{X}_{2}\right)$, i.e., all of (a) $R_{1} \geq$ $\bar{H}^{*}\left(X_{1} \mid X_{2}\right)$, (b) $R_{2} \geq \bar{H}^{*}\left(X_{2} \mid X_{1}\right)$, and (c) $R_{1}+R_{2} \geq$ $\bar{H}^{*}\left(X_{1}, X_{1}\right)$ are satisfied.

Hereinafter, we prove $R_{1} \geq \bar{H}^{*}\left(X_{1} \mid X_{2}\right)$ by contradiction. Assume that $R_{1}<\bar{H}^{*}\left(\boldsymbol{X}_{1} \mid \boldsymbol{X}_{2}\right)$ is satisfied. Then, there exists a constant $\gamma_{0}>0$ such that $R_{1} \leq \bar{H}^{*}\left(\boldsymbol{X}_{1} \mid \boldsymbol{X}_{2}\right)-3 \gamma_{0}$. In addition, we should note that $\bar{H}^{*}\left(\boldsymbol{X}_{1} \mid \boldsymbol{X}_{2}\right)$ can be expressed in the following form:

$$
\begin{aligned}
& \bar{H}^{*}\left(\boldsymbol{X}_{1} \mid \boldsymbol{X}_{2}\right)=\sup \{\beta: \\
& \left.\liminf _{n \rightarrow \infty} \operatorname{Pr}\left\{\frac{1}{n} \log \frac{1}{P_{X_{1}^{n} \mid X_{2}^{n}}\left(X_{1}^{n} \mid X_{2}^{n}\right)} \geq \beta\right\}>0\right\}
\end{aligned}
$$

(see [6, Appendix]). Therefore, there exist a constant $\delta_{0}>0$ and an integer $n_{0}$ such that

$$
\begin{aligned}
& \operatorname{Pr}\left\{\frac{1}{n} \log \frac{1}{P_{X_{1}^{n} \mid X_{2}^{n}}\left(X_{1}^{n} \mid X_{2}^{n}\right)} \geq \bar{H}^{*}\left(\boldsymbol{X}_{1} \mid \boldsymbol{X}_{2}\right)-\gamma_{0}\right\} \\
& \quad \geq 2 \delta_{0} \text { for all } n \geq n_{0} .
\end{aligned}
$$

Furthermore, Lemma 2 tells us that for all $n \geq 1$ any code $\left\{\left(\varphi_{n}^{(1)}, \varphi_{n}^{(2)}, \psi_{n}\right)\right\}_{n=1}^{\infty}$ satisfies (10) with $\gamma=\gamma_{0}$, which leads to

$$
\begin{gathered}
\varepsilon_{n} \geq \operatorname{Pr}\left\{\frac{1}{n} \log \frac{1}{P_{X_{1}^{n} \mid X_{2}^{n}}\left(X_{1}^{n} \mid X_{2}^{n}\right)} \geq \frac{1}{n} \log M_{n}^{(1)}+\gamma_{0}\right\} \\
-3 \cdot 2^{-n \gamma_{0}} \text { for all } n \geq 1 .
\end{gathered}
$$

Now, assume that $\left(R_{1}, R_{2}\right)$ is achievable in the optimistic sense. Then, for any $\gamma \in\left(0, \min \left\{\gamma_{0}, \delta_{0}\right\}\right)$ there exists a code $\left\{\left(\varphi_{n}^{(1)}, \varphi_{n}^{(2)}, \psi_{n}\right)\right\}_{n=1}^{\infty}$ with a subsequence $\left\{n_{i}\right\}_{i=1}^{\infty}$ satisfying $\frac{1}{n} \log M_{n}^{(1)} \leq R_{1}+\gamma \leq \bar{H}^{*}\left(\boldsymbol{X}_{1} \mid \boldsymbol{X}_{2}\right)-3 \gamma_{0}+\gamma \leq$ $\bar{H}^{*}\left(\boldsymbol{X}_{1} \mid \boldsymbol{X}_{2}\right)-2 \gamma_{0}$ and $\varepsilon_{n} \leq \gamma$ for all $n=n_{i}, i \geq 1$. Then, (13) leads to

$$
\begin{aligned}
\varepsilon_{n} \geq \operatorname{Pr}\{ & \left.\frac{1}{n} \log \frac{1}{P_{X_{1}^{n} \mid X_{2}^{n}}\left(X_{1}^{n} \mid X_{2}^{n}\right)} \geq \bar{H}^{*}\left(\boldsymbol{X}_{1} \mid \boldsymbol{X}_{2}\right)-\gamma_{0}\right\} \\
& -3 \cdot 2^{-n \gamma_{0}} \text { for all } n=n_{i}, i \geq 1,
\end{aligned}
$$

where the left side is less than $\gamma$ for all $n=n_{i}, i \geq 1$, and the right side is greater than $\delta_{0}(>\gamma)$ for all sufficiently large $n=n_{i}$ due to (12). This is a contradiction.

Since this argument can be applied to establishing (b) $R_{2} \geq \bar{H}^{*}\left(\boldsymbol{X}_{2} \mid \boldsymbol{X}_{1}\right)$ and (c) $R_{1}+R_{2} \geq \bar{H}^{*}\left(\boldsymbol{X}_{1}, \boldsymbol{X}_{1}\right)$ as well, we can conclude that $\left(R_{1}, R_{2}\right) \in \mathcal{S}^{*}\left(X_{1}, X_{2}\right)$ provided that $\left(R_{1}, R_{2}\right)$ is achievable in the optimistic sense. This establishes $\mathcal{R}_{\text {opt }}\left(\boldsymbol{X}_{1}, \boldsymbol{X}_{2}\right) \subset \mathcal{S}^{*}\left(\boldsymbol{X}_{1}, \boldsymbol{X}_{2}\right)$ because $\mathcal{S}^{*}\left(\boldsymbol{X}_{1}, \boldsymbol{X}_{2}\right)$ includes the boundaries.

\section{Discussions}

\subsection{Characterization of $\mathcal{R}\left(\boldsymbol{X}_{1}, \boldsymbol{X}_{2}\right)$}

So far, we have established an inner and an outer bounds 
of $\mathcal{R}_{\text {opt }}\left(\boldsymbol{X}_{1}, \boldsymbol{X}_{2}\right)$. In this subsection we revisit $\mathcal{R}\left(\boldsymbol{X}_{1}, \boldsymbol{X}_{2}\right)$ in Definition 1 and characterize $\mathcal{R}\left(\boldsymbol{X}_{1}, \boldsymbol{X}_{2}\right)$ in the same manner as Theorem 2.

To this end, define $\tilde{\mathcal{A}}\left(\boldsymbol{X}_{1}, \boldsymbol{X}_{2}\right)$ and $\tilde{\mathcal{B}}\left(\boldsymbol{X}_{1}, \boldsymbol{X}_{2}\right)$ by replacing the limit inferiors in (6) and (7) with the limit superiors, respectively. Define $\mathcal{W}\left(\boldsymbol{X}_{1}, \boldsymbol{X}_{2}\right)$ by replacing the limit inferior in (5) with the limit superior. Then, it is easily verified that $\mathcal{R}\left(\boldsymbol{X}_{1}, \boldsymbol{X}_{2}\right)$ satisfies

$$
\tilde{\mathcal{A}}\left(\boldsymbol{X}_{1}, \boldsymbol{X}_{2}\right) \subset \mathcal{W}^{\dagger}\left(\boldsymbol{X}_{1}, \boldsymbol{X}_{2}\right) \subset \mathcal{R}\left(\boldsymbol{X}_{1}, \boldsymbol{X}_{2}\right) \subset \tilde{\mathcal{B}}\left(\boldsymbol{X}_{1}, \boldsymbol{X}_{2}\right)
$$

similarly to the proof of Theorem 2 , where $\boldsymbol{W}^{\dagger}\left(\boldsymbol{X}_{1}, \boldsymbol{X}_{2}\right)$ denotes the closure of the complement of $\mathcal{W}\left(\boldsymbol{X}_{1}, \boldsymbol{X}_{2}\right)$. We can also verify that $\tilde{\mathcal{B}}\left(\boldsymbol{X}_{1}, \boldsymbol{X}_{2}\right)$ is expressed as $\mathcal{S}\left(\boldsymbol{X}_{1}, \boldsymbol{X}_{2}\right)$ in Theorem 1 similarly to Proposition 2.

In the ordinary case, however, we can prove $\tilde{\mathcal{B}}\left(\boldsymbol{X}_{1}, \boldsymbol{X}_{2}\right)$ $\subset \tilde{\mathcal{A}}\left(\boldsymbol{X}_{1}, \boldsymbol{X}_{2}\right)$ as well and therefore $\mathcal{W}^{\dagger}\left(\boldsymbol{X}_{1}, \boldsymbol{X}_{2}\right)=\mathcal{R}\left(\boldsymbol{X}_{1}\right.$, $\left.\boldsymbol{X}_{2}\right)=\mathcal{S}\left(\boldsymbol{X}_{1}, \boldsymbol{X}_{2}\right)$ without any assumption on $\left(\boldsymbol{X}_{1}, \boldsymbol{X}_{2}\right)$. This explains a reason why $\mathcal{R}\left(\boldsymbol{X}_{1}, \boldsymbol{X}_{2}\right)$ is expressed in a closed form and is coincident with $\mathcal{S}\left(\boldsymbol{X}_{1}, \boldsymbol{X}_{2}\right)$.

In order to verify $\tilde{\mathcal{B}}\left(\boldsymbol{X}_{1}, \boldsymbol{X}_{2}\right) \subset \tilde{\mathcal{A}}\left(\boldsymbol{X}_{1}, \boldsymbol{X}_{2}\right)$, let $\left(R_{1}, R_{2}\right)$ be an arbitrary element of $\tilde{\mathcal{B}}\left(\boldsymbol{X}_{1}, \boldsymbol{X}_{2}\right)$. By the definition of $\tilde{\mathcal{B}}\left(\boldsymbol{X}_{1}, \boldsymbol{X}_{2}\right)$, it holds that $\lim \sup _{n \rightarrow \infty} \operatorname{Pr}\left\{E_{n}^{(k)}\left(R_{k}+\gamma\right)\right\}=0$ for $k=1,2$ and $\limsup _{n \rightarrow \infty} \operatorname{Pr}\left\{E_{n}^{(3)}\left(R_{1}+R_{2}+\gamma\right)\right\}=0$. Then, we have

$$
\begin{aligned}
& \limsup _{n \rightarrow \infty} \operatorname{Pr}\left\{E_{n}^{(1)}\left(R_{1}+\gamma\right) \cup E_{n}^{(2)}\left(R_{2}+\gamma\right) \cup E_{n}^{(3)}\left(R_{1}+R_{2}+\gamma\right)\right\} \\
& \leq \underset{n \rightarrow \infty}{\lim \sup } \operatorname{Pr}\left\{E_{n}^{(1)}\left(R_{1}+\gamma\right)\right\}+\limsup _{n \rightarrow \infty} \operatorname{Pr}\left\{E_{n}^{(2)}\left(R_{2}+\gamma\right)\right\} \\
& +\limsup \operatorname{Pr}\left\{E_{n}^{(3)}\left(R_{1}+R_{2}+\gamma\right)\right\} \\
& =0 \text {, }
\end{aligned}
$$

where the inequality follows from the union bound and $\limsup \sup _{n \rightarrow \infty}\left(a_{n}+b_{n}\right) \leq \lim \sup _{n \rightarrow \infty} a_{n}+\limsup _{n \rightarrow \infty} b_{n}$ for any real-valued sequences $\left\{a_{n}\right\}_{n=1}^{\infty}$ and $\left\{b_{n}\right\}_{n=1}^{\infty}$. Since the left side is nonnegative, (15) implies that $\left(R_{1}, R_{2}\right) \in \tilde{\mathcal{A}}\left(\boldsymbol{X}_{1}, \boldsymbol{X}_{2}\right)$. This establishes $\tilde{\mathcal{B}}\left(\boldsymbol{X}_{1}, \boldsymbol{X}_{2}\right) \subset \tilde{\mathcal{A}}\left(\boldsymbol{X}_{1}, \boldsymbol{X}_{2}\right)$.

Notice that we cannot use the same argument to establish $\mathcal{B}\left(\boldsymbol{X}_{1}, \boldsymbol{X}_{2}\right) \subset \mathcal{A}\left(\boldsymbol{X}_{1}, \boldsymbol{X}_{2}\right)$ because only the opposite side the inequality holds with respect to the limit inferior. In Example 3 in Sect. 4.2 we will see an example of $\left(\boldsymbol{X}_{1}, \boldsymbol{X}_{2}\right)$ satisfying $\mathcal{A}\left(\boldsymbol{X}_{1}, \boldsymbol{X}_{2}\right) \subsetneq \mathcal{B}\left(\boldsymbol{X}_{1}, \boldsymbol{X}_{2}\right)$.

\subsection{Sufficient Conditions for $\mathcal{A}\left(\boldsymbol{X}_{1}, \boldsymbol{X}_{2}\right)=\mathcal{B}\left(\boldsymbol{X}_{1}, \boldsymbol{X}_{2}\right)$}

In this subsection, we investigate sufficient conditions on $\left(\boldsymbol{X}_{1}, \boldsymbol{X}_{2}\right)$ under which $\mathcal{A}\left(\boldsymbol{X}_{1}, \boldsymbol{X}_{2}\right)=\mathcal{B}\left(\boldsymbol{X}_{1}, \boldsymbol{X}_{2}\right)$ holds. Theorem 2 and Proposition 2 guarantee that $\mathcal{R}_{\mathrm{opt}}\left(\boldsymbol{X}_{1}, \boldsymbol{X}_{2}\right)=$ $\mathcal{U}^{\dagger}\left(\boldsymbol{X}_{1}, \boldsymbol{X}_{2}\right)=\mathcal{S}^{*}\left(\boldsymbol{X}_{1}, \boldsymbol{X}_{2}\right)$ under such sufficient conditions.

We begin with the simple sufficient condition given in the following proposition. This sufficient condition is shown to be valid by using the argument given in Sect. 4.1.

Proposition 4: If $\bar{H}\left(\boldsymbol{X}_{1} \mid \boldsymbol{X}_{2}\right)=\bar{H}^{*}\left(\boldsymbol{X}_{1} \mid \boldsymbol{X}_{2}\right), \bar{H}\left(\boldsymbol{X}_{2} \mid \boldsymbol{X}_{1}\right)=$ $\bar{H}^{*}\left(\boldsymbol{X}_{2} \mid \boldsymbol{X}_{1}\right)$ and $\bar{H}\left(\boldsymbol{X}_{1}, \boldsymbol{X}_{2}\right)=\bar{H}^{*}\left(\boldsymbol{X}_{1}, \boldsymbol{X}_{2}\right)$, then it holds that $\mathcal{R}_{\mathrm{opt}}\left(\boldsymbol{X}_{1}, \boldsymbol{X}_{2}\right)=\mathcal{U}^{\dagger}\left(\boldsymbol{X}_{1}, \boldsymbol{X}_{2}\right)=\mathcal{S}^{*}\left(\boldsymbol{X}_{1}, \boldsymbol{X}_{2}\right)$.

Proof: Clearly, $\mathcal{S}\left(\boldsymbol{X}_{1}, \boldsymbol{X}_{2}\right)=\mathcal{S}^{*}\left(\boldsymbol{X}_{1}, \boldsymbol{X}_{2}\right)$ holds by the assumption. So far, we have already established that $\mathcal{B}\left(\boldsymbol{X}_{1}, \boldsymbol{X}_{2}\right)=\mathcal{S}^{*}\left(\boldsymbol{X}_{1}, \boldsymbol{X}_{2}\right)$ in Proposition 2 and $\tilde{\mathcal{B}}\left(\boldsymbol{X}_{1}, \boldsymbol{X}_{2}\right)=$ $\mathcal{S}\left(\boldsymbol{X}_{1}, \boldsymbol{X}_{2}\right)$ in Sect. 4.1. Hence, $\mathcal{S}\left(\boldsymbol{X}_{1}, \boldsymbol{X}_{2}\right)=\mathcal{S}^{*}\left(\boldsymbol{X}_{1}, \boldsymbol{X}_{2}\right)$ implies $\mathcal{B}\left(\boldsymbol{X}_{1}, \boldsymbol{X}_{2}\right)=\tilde{\mathcal{B}}\left(\boldsymbol{X}_{1}, \boldsymbol{X}_{2}\right)$. In addition, we should note $\tilde{\mathcal{A}}\left(\boldsymbol{X}_{1}, \boldsymbol{X}_{2}\right) \subset \mathcal{A}\left(\boldsymbol{X}_{1}, \boldsymbol{X}_{2}\right)$, which immediately follows from their definitions. Then, in view of Theorem 2 it holds that

$$
\begin{aligned}
& \tilde{\mathcal{A}}\left(\boldsymbol{X}_{1}, \boldsymbol{X}_{2}\right) \subset \mathcal{A}\left(\boldsymbol{X}_{1}, \boldsymbol{X}_{2}\right) \subset \mathcal{U}^{\dagger}\left(\boldsymbol{X}_{1}, \boldsymbol{X}_{2}\right) \\
& \quad \subset \mathcal{R}_{\text {opt }}\left(\boldsymbol{X}_{1}, \boldsymbol{X}_{2}\right) \subset \mathcal{B}\left(\boldsymbol{X}_{1}, \boldsymbol{X}_{2}\right)=\tilde{\mathcal{B}}\left(\boldsymbol{X}_{1}, \boldsymbol{X}_{2}\right) .
\end{aligned}
$$

Since we have proved that $\tilde{\mathcal{A}}\left(\boldsymbol{X}_{1}, \boldsymbol{X}_{2}\right)=\tilde{\mathcal{B}}\left(\boldsymbol{X}_{1}, \boldsymbol{X}_{2}\right)$ in Sect. 4.1, the claim in this proposition follows.

Example 1: Let $P_{X_{1} X_{2}}$ be a joint probability distribution on $X_{1} \times X_{2}$ satisfying $H\left(X_{1} X_{2}\right)<\infty$, where $H\left(X_{1} X_{2}\right)$ denotes the joint entropy of $X_{1}$ and $X_{2}$. Let $X_{1}$ and $\boldsymbol{X}_{2}$ be two correlated stationary memoryless sources induced by $P_{X_{1} X_{2}}$. Clearly, by the weak law of large numbers we have $\bar{H}\left(\boldsymbol{X}_{1} \mid \boldsymbol{X}_{2}\right)=\bar{H}^{*}\left(\boldsymbol{X}_{1} \mid \boldsymbol{X}_{2}\right)=H\left(X_{1} \mid X_{2}\right), \bar{H}\left(\boldsymbol{X}_{2} \mid \boldsymbol{X}_{1}\right)=$ $\bar{H}^{*}\left(\boldsymbol{X}_{2} \mid \boldsymbol{X}_{1}\right)=H\left(X_{2} \mid X_{1}\right)$ and $\bar{H}\left(\boldsymbol{X}_{1}, \boldsymbol{X}_{2}\right)=\bar{H}^{*}\left(\boldsymbol{X}_{1}, \boldsymbol{X}_{2}\right)=$ $H\left(X_{1} X_{2}\right)$ for these sources, where $H\left(X_{k} \mid X_{l}\right)$ denotes the conditional entropy of $X_{k}$ given $X_{l}$ for $(k, l)=(1,2)$ and $(2,1)$. Hence, Proposition 4 guarantees that

$$
\begin{gathered}
\mathcal{R}\left(\boldsymbol{X}_{1}, \boldsymbol{X}_{2}\right)=\mathcal{R}_{\mathrm{opt}}\left(\boldsymbol{X}_{1}, \boldsymbol{X}_{2}\right) \\
=\left\{\left(R_{1}, R_{2}\right): R_{1} \geq H\left(X_{1} \mid X_{2}\right), R_{2} \geq H\left(X_{2} \mid X_{1}\right)\right. \\
\text { and } \left.R_{1}+R_{2} \geq H\left(X_{1} X_{2}\right)\right\} .
\end{gathered}
$$

Next, we introduce a new class of correlated sources.

Definition 4: Let $\boldsymbol{X}_{1}$ and $\boldsymbol{X}_{2}$ be two correlated general sources. We say that $\left(\boldsymbol{X}_{1}, \boldsymbol{X}_{2}\right)$ is synchronizing if for any $\gamma>0$ and $\eta \in(0,1)$ there exists a subsequence $\left\{n_{i}\right\}_{i=1}^{\infty}$ such that all of

$$
\begin{aligned}
& \operatorname{Pr}\left\{\frac{1}{n} \log \frac{1}{P_{X_{1}^{n} \mid X_{2}^{n}}\left(X_{1}^{n} \mid X_{2}^{n}\right)} \geq \bar{H}^{*}\left(\boldsymbol{X}_{1} \mid \boldsymbol{X}_{2}\right)+\gamma\right\} \leq \eta, \\
& \operatorname{Pr}\left\{\frac{1}{n} \log \frac{1}{P_{X_{2}^{n} \mid X_{1}^{n}}\left(X_{2}^{n} \mid X_{1}^{n}\right)} \geq \bar{H}^{*}\left(\boldsymbol{X}_{2} \mid \boldsymbol{X}_{1}\right)+\gamma\right\} \leq \eta, \\
& \operatorname{Pr}\left\{\frac{1}{n} \log \frac{1}{P_{X_{1}^{n} X_{2}^{n}}\left(X_{1}^{n}, X_{2}^{n}\right)} \geq \bar{H}^{*}\left(\boldsymbol{X}_{1}, \boldsymbol{X}_{2}\right)+\gamma\right\} \leq \eta
\end{aligned}
$$

are satisfied for all $n=n_{i}, i \geq 1$.

Note that owing to the definitions of $\bar{H}^{*}\left(\boldsymbol{X}_{1} \mid \boldsymbol{X}_{2}\right)$, $\bar{H}^{*}\left(\boldsymbol{X}_{2} \mid \boldsymbol{X}_{1}\right)$ and $\bar{H}^{*}\left(\boldsymbol{X}_{1}, \boldsymbol{X}_{2}\right)$, each of the above three inequalities in Definition 4 is satisfied infinitely often. The synchronizing property in Definition 4 actually requires that all the three inequalities are satisfied simultaneously with the same subsequence $\left\{n_{i}\right\}_{i=1}^{\infty}$.

The following proposition indicates that the synchronizing property of $\left(\boldsymbol{X}_{1}, \boldsymbol{X}_{2}\right)$ is another sufficient condition for $\mathcal{A}\left(\boldsymbol{X}_{1}, \boldsymbol{X}_{2}\right)=\mathcal{B}\left(\boldsymbol{X}_{1}, \boldsymbol{X}_{2}\right)$.

Proposition 5: If $\left(\boldsymbol{X}_{1}, \boldsymbol{X}_{2}\right)$ is synchronizing, $\mathcal{R}_{\mathrm{opt}}\left(\boldsymbol{X}_{1}, \boldsymbol{X}_{2}\right)$ $=\mathcal{U}^{\dagger}\left(X_{1}, X_{2}\right)=\mathcal{S}^{*}\left(X_{1}, X_{2}\right)$.

Proof: In view of Theorem 2, it suffices to establish 
$\mathcal{B}\left(\boldsymbol{X}_{1}, \boldsymbol{X}_{2}\right) \subset \mathcal{A}\left(\boldsymbol{X}_{1}, \boldsymbol{X}_{2}\right)$. Fix $\left(R_{1}, R_{2}\right) \in \mathcal{B}\left(\boldsymbol{X}_{1}, \boldsymbol{X}_{2}\right)$ arbitrarily. Then, Proposition 2 tells us that all of $R_{1} \geq \bar{H}^{*}\left(X_{1} \mid X_{2}\right)$, $R_{2} \geq \bar{H}^{*}\left(\boldsymbol{X}_{2} \mid \boldsymbol{X}_{1}\right)$ and $R_{1}+R_{2} \geq \bar{H}^{*}\left(\boldsymbol{X}_{1}, \boldsymbol{X}_{2}\right)$ are satisfied. Since $\left(\boldsymbol{X}_{1}, \boldsymbol{X}_{2}\right)$ is assumed to be synchronizing, for any constants $\gamma>0$ and $\eta \in(0,1)$ there exists a subsequence $\left\{n_{i}\right\}_{i=1}^{\infty}$ satisfying

$$
\begin{aligned}
\operatorname{Pr} & \left\{E_{n}^{(1)}\left(R_{1}+\gamma\right)\right\} \\
& =\operatorname{Pr}\left\{\frac{1}{n} \log \frac{1}{P_{X_{1}^{n} \mid X_{2}^{n}}\left(X_{1}^{n} \mid X_{2}^{n}\right)} \geq R_{1}+\gamma\right\} \\
& \leq \operatorname{Pr}\left\{\frac{1}{n} \log \frac{1}{P_{X_{1}{ }^{n} \mid X_{2}{ }^{n}}\left(X_{1}{ }^{n} \mid X_{2}{ }^{n}\right)} \geq \bar{H}^{*}\left(X_{1} \mid X_{2}\right)+\gamma\right\} \\
& \leq \eta \quad \text { for all } n=n_{i} \text { and } i \geq 1 .
\end{aligned}
$$

Similarly, we have $\operatorname{Pr}\left\{E_{n}^{(2)}\left(R_{2}+\gamma\right)\right\} \leq \eta$ and $\operatorname{Pr}\left\{E_{n}^{(3)}\left(R_{1}+R_{2}+\right.\right.$ $\gamma)\} \leq \eta$ for all $n=n_{i}, i \geq 1$. Therefore, it holds that

$$
\begin{aligned}
\operatorname{Pr} & \left.E_{n}^{(1)}\left(R_{1}+\gamma\right) \cup E_{n}^{(2)}\left(R_{2}+\gamma\right) \cup E_{n}^{(3)}\left(R_{1}+R_{2}+\gamma\right)\right\} \\
\leq & \operatorname{Pr}\left\{E_{n}^{(1)}\left(R_{1}+\gamma\right)\right\}+\operatorname{Pr}\left\{E_{n}^{(2)}\left(R_{2}+\gamma\right)\right\} \\
& +\operatorname{Pr}\left\{E_{n}^{(3)}\left(R_{1}+R_{2}+\gamma\right)\right\} \\
\leq & 3 \eta \quad \text { for all } n=n_{i} \text { and } i \geq 1,
\end{aligned}
$$

which means that the limit inferior of the left side is equal to zero. Since $\left(R_{1}, R_{2}\right) \in \mathcal{B}\left(\boldsymbol{X}_{1}, \boldsymbol{X}_{2}\right)$ is arbitrary, $\mathcal{B}\left(\boldsymbol{X}_{1}, \boldsymbol{X}_{2}\right) \subset$ $\mathcal{A}\left(\boldsymbol{X}_{1}, \boldsymbol{X}_{2}\right)$ follows.

The notion of synchronizing property in Definition 4 leads to an interesting implication on inner bounds of $\mathcal{R}_{\text {opt }}\left(\boldsymbol{X}_{1}, \boldsymbol{X}_{2}\right)$. Assume that for any $\gamma>0$ there exists a subsequence $\left\{n_{i}\right\}_{i=1}^{\infty}$ such that (17) and (19) hold for all $n=n_{i}, i \geq 1$. Then, we can show that

$$
\begin{aligned}
\mathcal{V}_{1}\left(\boldsymbol{X}_{1}, \boldsymbol{X}_{2}\right)=\left\{\left(R_{1}, R_{2}\right): R_{1} \geq \bar{H}^{*}\left(\boldsymbol{X}_{1} \mid \boldsymbol{X}_{2}\right),\right. \\
\left.R_{2} \geq \bar{H}\left(\boldsymbol{X}_{2} \mid \boldsymbol{X}_{1}\right) \text { and } R_{1}+R_{2} \geq \bar{H}^{*}\left(\boldsymbol{X}_{1}, \boldsymbol{X}_{2}\right)\right\}
\end{aligned}
$$

is a subset of $\mathcal{R}_{\text {opt }}\left(\boldsymbol{X}_{1}, \boldsymbol{X}_{2}\right)$. This fact is verified by checking $\mathcal{V}_{1}\left(\boldsymbol{X}_{1}, \boldsymbol{X}_{2}\right) \subset \mathcal{A}\left(\boldsymbol{X}_{1}, \boldsymbol{X}_{2}\right)$ as follows. Fix $\left(R_{1}, R_{2}\right) \subset$ $\mathcal{V}_{1}\left(\boldsymbol{X}_{1}, \boldsymbol{X}_{2}\right)$ arbitrarily. Then, we have

$$
\begin{gathered}
\operatorname{Pr}\left\{E_{n}^{(1)}\left(R_{1}+\gamma\right)\right\}+\operatorname{Pr}\left\{E_{n}^{(3)}\left(R_{1}+R_{2}+\gamma\right)\right\} \leq 2 \eta \\
\text { for all } n=n_{i} \text { and } i \geq 1, \text { and }
\end{gathered}
$$

$\operatorname{Pr}\left\{E_{n}^{(2)}\left(R_{2}+\gamma\right)\right\} \leq \eta \quad$ for all sufficiently large $n$.

Thus, there exists an integer $i_{0}$ such that

$$
\begin{gathered}
\operatorname{Pr}\left\{E_{n}^{(1)}\left(R_{1}+\gamma\right) \cup E_{n}^{(2)}\left(R_{2}+\gamma\right) \cup E_{n}^{(3)}\left(R_{1}+R_{2}+\gamma\right)\right\} \leq 3 \eta \\
\text { for all } n=n_{i} \text { and } i \geq i_{0},
\end{gathered}
$$

which means the limit inferior of the left side is equal to zero. On the other hand, we can similarly prove that

$$
\begin{aligned}
& \mathcal{V}_{2}\left(\boldsymbol{X}_{1}, \boldsymbol{X}_{2}\right)=\left\{\left(R_{1}, R_{2}\right): R_{1} \geq \bar{H}\left(\boldsymbol{X}_{1} \mid \boldsymbol{X}_{2}\right),\right. \\
& \left.R_{2} \geq \bar{H}^{*}\left(\boldsymbol{X}_{2} \mid \boldsymbol{X}_{1}\right) \text { and } R_{1}+R_{2} \geq \bar{H}\left(\boldsymbol{X}_{1}, \boldsymbol{X}_{2}\right)\right\}
\end{aligned}
$$

is a subset of $\mathcal{A}\left(\boldsymbol{X}_{1}, \boldsymbol{X}_{2}\right)$ with no assumption on $\left(\boldsymbol{X}_{1}, \boldsymbol{X}_{2}\right)$. Example 2: Let $P_{X_{1} X_{2}}^{(1)}$ and $P_{X_{1} X_{2}}^{(2)}$ be the joint probability distributions on $X_{1} \times X_{2}$ satisfying $H\left(X_{1}^{(1)} \mid X_{2}^{(1)}\right) \leq H\left(X_{1}^{(2)} \mid X_{2}^{(2)}\right)$, $H\left(X_{2}^{(1)} \mid X_{1}^{(1)}\right) \leq H\left(X_{2}^{(2)} \mid X_{1}^{(2)}\right)$ and $H\left(X_{1}^{(1)} X_{2}^{(1)}\right) \leq H\left(X_{1}^{(2)} X_{2}^{(2)}\right)<$ $\infty$. Define $\left(\boldsymbol{X}_{1}, \boldsymbol{X}_{2}\right)=\left\{\left(X_{1}^{n}, X_{2}^{n}\right)\right\}_{n=1}^{\infty}$ as the pair of general sources satisfying

$$
P_{X_{1}^{n} X_{2}^{n}}\left(x_{1}^{n}, \tilde{x}_{1}^{n}\right)= \begin{cases}\prod_{i=1}^{n} P_{X_{1} X_{2}}^{(1)}\left(x_{i}, \tilde{x}_{i}\right), & \text { if } n \text { is odd, } \\ \prod_{i=1}^{n} P_{X_{1} X_{2}}^{(2)}\left(x_{i}, \tilde{x}_{i}\right), & \text { if } n \text { is even, }\end{cases}
$$

where $x_{1}^{n}=\left(x_{1}, \ldots, x_{2}\right)$ and $\tilde{x}_{1}^{n}=\left(\tilde{x}_{1}, \ldots, \tilde{x}_{n}\right)$. Since it holds that $\bar{H}\left(\boldsymbol{X}_{1} \mid \boldsymbol{X}_{2}\right)=H\left(X_{1}^{(2)} \mid X_{2}^{(2)}\right), \bar{H}\left(\boldsymbol{X}_{2} \mid \boldsymbol{X}_{1}\right)=H\left(X_{2}^{(2)} \mid X_{1}^{(2)}\right)$, and $\bar{H}\left(\boldsymbol{X}_{1} \boldsymbol{X}_{2}\right)=H\left(X_{1}^{(2)} X_{2}^{(2)}\right)$, we have

$$
\begin{aligned}
& \mathcal{R}\left(X_{1}, X_{2}\right)=\left\{\left(R_{1}, R_{2}\right): R_{1} \geq H\left(X_{1}^{(2)} \mid X_{2}^{(2)}\right),\right. \\
& \left.\quad R_{2} \geq H\left(X_{2}^{(2)} \mid X_{1}^{(2)}\right) \text { and } R_{1}+R_{2} \geq H\left(X_{1}^{(2)} \mid X_{2}^{(2)}\right)\right\} .
\end{aligned}
$$

In addition, since $\left(\boldsymbol{X}_{1}, \boldsymbol{X}_{2}\right)$ is synchronizing and satisfies $\bar{H}^{*}\left(\boldsymbol{X}_{1} \mid \boldsymbol{X}_{2}\right)=H\left(X_{1}^{(1)} \mid X_{2}^{(1)}\right), \bar{H}^{*}\left(\boldsymbol{X}_{2} \mid \boldsymbol{X}_{1}\right)=H\left(X_{2}^{(1)} \mid X_{1}^{(1)}\right)$, and $\bar{H}^{*}\left(\boldsymbol{X}_{1} \boldsymbol{X}_{2}\right)=H\left(X_{1}^{(1)} X_{2}^{(1)}\right)$, we have

$$
\begin{gathered}
\mathcal{R}_{\mathrm{opt}}\left(X_{1}, X_{2}\right)=\left\{\left(R_{1}, R_{2}\right): R_{1} \geq H\left(X_{1}^{(1)} \mid X_{2}^{(1)}\right),\right. \\
\left.R_{2} \geq H\left(X_{2}^{(1)} \mid X_{1}^{(1)}\right) \text { and } R_{1}+R_{2} \geq H\left(X_{1}^{(1)} X_{2}^{(1)}\right)\right\} .
\end{gathered}
$$

Figure 2 shows $\mathcal{R}\left(\boldsymbol{X}_{1}, \boldsymbol{X}_{2}\right)$ and $\mathcal{R}_{\text {opt }}\left(\boldsymbol{X}_{1}, \boldsymbol{X}_{2}\right)$ in this example. Example 3: $\quad$ Suppose that $P_{X_{1} X_{2}}^{(1)}$ and $P_{X_{1} X_{2}}^{(2)}$ satisfy $H\left(X_{1}^{(1)} \mid X_{2}^{(1)}\right) \leq H\left(X_{1}^{(2)} \mid X_{2}^{(2)}\right), H\left(X_{1}^{(1)} X_{2}^{(1)}\right) \leq H\left(X_{1}^{(2)} X_{2}^{(2)}\right)<\infty$, and $H\left(X_{2}^{(2)} \mid X_{1}^{(2)}\right) \leq H\left(X_{2}^{(1)} \mid X_{1}^{(1)}\right)$ in Example 2. In this case, we can easily verify from Theorems 1 and 2 that

$$
\begin{aligned}
& \mathcal{R}\left(X_{1}, X_{2}\right)=\left\{\left(R_{1}, R_{2}\right): R_{1} \geq H\left(X_{1}^{(2)} \mid X_{2}^{(2)}\right),\right. \\
& \left.R_{2} \geq H\left(X_{2}^{(1)} \mid X_{1}^{(1)}\right) \text { and } R_{1}+R_{2} \geq H\left(X_{1}^{(2)} X_{2}^{(2)}\right)\right\}
\end{aligned}
$$

and

$$
\mathcal{V}\left(\boldsymbol{X}_{1}, \boldsymbol{X}_{2}\right) \subset \mathcal{R}_{\mathrm{opt}}\left(\boldsymbol{X}_{1}, \boldsymbol{X}_{2}\right) \subset \mathcal{S}^{*}\left(\boldsymbol{X}_{1}, \boldsymbol{X}_{2}\right),
$$

where $\mathcal{V}\left(\boldsymbol{X}_{1}, \boldsymbol{X}_{2}\right)=\mathcal{V}_{1}\left(\boldsymbol{X}_{1}, \boldsymbol{X}_{2}\right) \cup \mathcal{V}_{2}\left(\boldsymbol{X}_{1}, \boldsymbol{X}_{2}\right)$,

$$
\begin{aligned}
& \mathcal{V}_{i}\left(X_{1}, X_{2}\right)=\left\{\left(R_{1}, R_{2}\right): R_{1} \geq H\left(X_{1}^{(i)} \mid X_{2}^{(i)}\right),\right. \\
& \left.R_{2} \geq H\left(X_{2}^{(i)} \mid X_{1}^{(i)}\right) \text { and } R_{1}+R_{2} \geq H\left(X_{1}^{(i)} X_{2}^{(i)}\right)\right\}
\end{aligned}
$$

for $i=1,2$ and

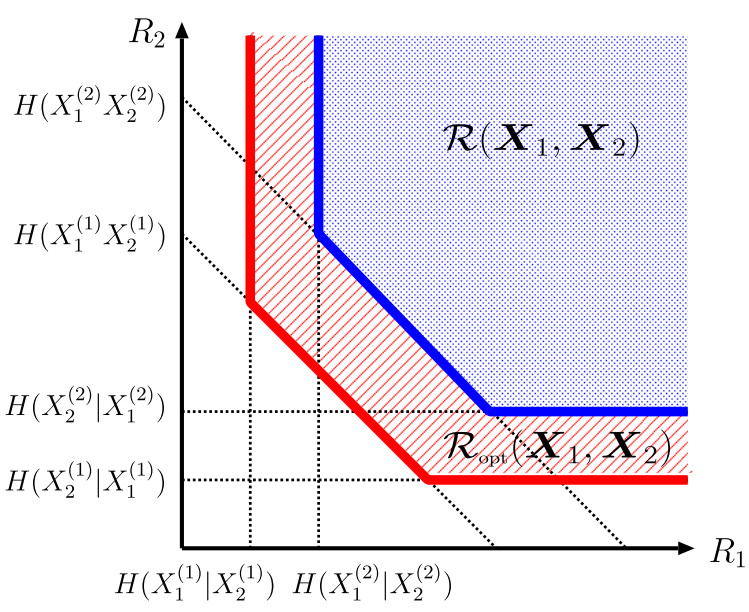

Fig. $2 \mathcal{R}\left(\boldsymbol{X}_{1}, \boldsymbol{X}_{2}\right)$ and $\mathcal{R}_{\text {opt }}\left(\boldsymbol{X}_{1}, \boldsymbol{X}_{2}\right)$ in Example 2 . 


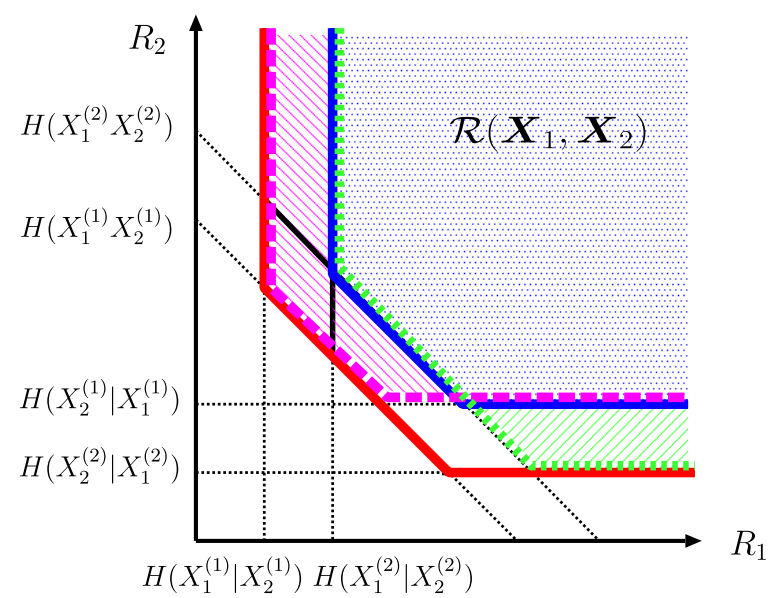

Fig. $3 \mathcal{S}^{*}\left(\boldsymbol{X}_{1}, \boldsymbol{X}_{2}\right), \mathcal{V}\left(\boldsymbol{X}_{1}, \boldsymbol{X}_{2}\right)$ and $\mathcal{R}\left(\boldsymbol{X}_{1}, \boldsymbol{X}_{2}\right)$ in Example 3. The broken (purple) line and the dotted (green) line correspond to the boundaries of $\mathcal{V}_{1}\left(\boldsymbol{X}_{1}, \boldsymbol{X}_{2}\right)$ and $\mathcal{V}_{2}\left(\boldsymbol{X}_{1}, \boldsymbol{X}_{2}\right)$, respectively.

$$
\begin{aligned}
& \mathcal{S}^{*}\left(X_{1}, X_{2}\right)=\left\{\left(R_{1}, R_{2}\right): R_{1} \geq H\left(X_{1}^{(1)} \mid X_{2}^{(1)}\right),\right. \\
& \left.\quad R_{2} \geq H\left(X_{2}^{(2)} \mid X_{1}^{(2)}\right) \text { and } R_{1}+R_{2} \geq H\left(X_{1}^{(1)} X_{2}^{(1)}\right)\right\} .
\end{aligned}
$$

Figure 3 shows the relationship of $\mathcal{S}^{*}\left(\boldsymbol{X}_{1}, \boldsymbol{X}_{2}\right), \mathcal{V}\left(\boldsymbol{X}_{1}, \boldsymbol{X}_{2}\right)$ and $\mathcal{R}\left(\boldsymbol{X}_{1}, \boldsymbol{X}_{2}\right)$ in this example.

In Example 3, we can actually prove that $\mathcal{R}_{\mathrm{opt}}\left(\boldsymbol{X}_{1}\right.$, $\left.\boldsymbol{X}_{2}\right)=\mathcal{V}\left(\boldsymbol{X}_{1}, \boldsymbol{X}_{2}\right)$ by using a contradiction argument. Suppose that $\left(R_{1}, R_{2}\right) \in \mathcal{S}^{*}\left(X_{1}, X_{2}\right) \backslash \mathcal{V}\left(X_{1}, X_{2}\right)$ is achievable in the optimistic sense. Then, there exists a code $\left\{\left(\varphi_{n}^{(1)}, \varphi_{n}^{(2)}, \psi_{n}\right)\right\}_{n=1}^{\infty}$ satisfying $\frac{1}{n} \log M_{n}^{(k)} \leq R_{k}+\gamma, k=1,2$, and $\varepsilon_{n} \leq \gamma$ for any $\gamma>0$ with some subsequence $\left\{n_{i}\right\}_{i=1}^{\infty}$. We first note that we can choose a sufficiently small $\gamma_{0} \in\left(0, \frac{1}{3}\right)$ such that $\left(R_{1}+2 \gamma_{0}, R_{2}+2 \gamma_{0}\right) \in \mathcal{S}^{*}\left(\boldsymbol{X}_{1}, \boldsymbol{X}_{2}\right) \backslash \mathcal{V}\left(\boldsymbol{X}_{1}, \boldsymbol{X}_{2}\right)$. Then, the code $\left\{\left(\varphi_{n}^{(1)}, \varphi_{n}^{(2)}, \psi_{n}\right)\right\}_{n=1}^{\infty}$ satisfies $\frac{1}{n} \log M_{n}^{(k)} \leq$ $R_{k}+\gamma_{0}, k=1,2$, and $\varepsilon_{n} \leq \gamma_{0}$ for a subsequence $\left\{n_{i}\right\}_{i=1}^{\infty}$. However, since $\left(R_{1}, R_{2}\right) \in \mathcal{S}^{*}\left(\boldsymbol{X}_{1}, \boldsymbol{X}_{2}\right) \backslash \mathcal{V}\left(\boldsymbol{X}_{1}, \boldsymbol{X}_{2}\right)$ implies that $R_{2}+\gamma_{0} \leq H\left(X_{2}^{(1)} \mid X_{1}^{(1)}\right)-\gamma_{0}$ and $R_{1}+R_{2}+\gamma_{0} \leq$ $H\left(X_{1}^{(2)} X_{2}^{(2)}\right)-\gamma_{0}$, due to the weak law of large numbers it holds that $\operatorname{Pr}\left\{E_{n}^{(2)}\left(R_{2}+\gamma_{0}\right)\right\} \geq 1-\gamma_{0}$ for all sufficiently large odd $n$ and $\operatorname{Pr}\left\{E_{n}^{(3)}\left(R_{1}+R_{2}+\gamma_{0}\right)\right\} \geq 1-\gamma_{0}$ for all sufficiently large even $n$, which yields $\operatorname{Pr}\left\{E_{n}^{(1)}\left(R_{1}+\gamma_{0}\right) \cup E_{n}^{(2)}\left(R_{1}+\gamma_{0}\right) \cup\right.$ $\left.E_{n}^{(3)}\left(R_{1}+R_{2}+\gamma_{0}\right)\right\} \geq 1-\gamma_{0}$ for all sufficiently large $n$. Then, we can prove that $\varepsilon_{n} \geq 1-\gamma_{0}-3 \cdot 2^{-n \gamma_{0}} \geq 1-2 \gamma_{0}$ for all sufficiently large $n$ similarly to (13) and (14) by using Lemma 2. This contradicts $\varepsilon_{n} \leq \gamma_{0}$ for all $n=n_{i}, i \geq 1$, because $\gamma_{0} \in\left(0, \frac{1}{3}\right)$.

\section{Conclusion}

In this paper the achievable rate region $\mathcal{R}_{\mathrm{opt}}\left(\boldsymbol{X}_{1}, \boldsymbol{X}_{2}\right)$ in the optimistic sense for separate coding of two general sources $\left(\boldsymbol{X}_{1}, \boldsymbol{X}_{2}\right)$ is discussed. We have given an inner and an outer bounds of $\mathcal{R}_{\mathrm{opt}}\left(\boldsymbol{X}_{1}, \boldsymbol{X}_{2}\right)$, where the outer bound is described by using new information-theoretic quantities. We have clarified two sufficient conditions under which the inner bound coincides with the outer bounds.

We conclude this paper by pointing out that extension of the obtained results to the case of $m(\geq 2)$ correlated general sources is straightforward.

\section{Acknowledgement}

The author would like to thank anonymous referees for their comments to improve the quality of this paper.

\section{References}

[1] P.-N. Chen and F. Alajaji, "Optimistic Shannon coding theorems for arbitrary single-user systems," IEEE Trans. Inf. Theory, vol.45, no.7, pp.2623-2629, 1999.

[2] T.M. Cover, "A proof of the data compression theorem of Slepian and Wolf for ergodic sources," IEEE Trans. Inf. Theory, vol.IT-22, no.2, pp.226-228, 1975.

[3] T.S. Han and S. Verdú, "Approximation theory of output statistics," IEEE Trans. Inf. Theory, vol.39, no.3, pp.752-772, 1993.

[4] T.S. Han, Information-Spectrum Methods in Information Theory, Springer, 2003.

[5] M. Hayashi, "Second order asymptotics in fixed-length source coding and intrinsic randomness," IEEE Trans. Inf. Theory, vol.54, no.10, pp.4619-4637, 2008.

[6] H. Koga, "Four limits in probability and coding of a general source," IEICE Trans. Fundamentals, vol.E94-A, no.11, pp.2073-2082, Nov. 2011.

[7] H. Koga, "On the achievable rate region in the optimistic sense for independent coding of two correlated general sources," IEICE Technical Report, IT2011-32, 2011.

[8] S. Miyake and F. Kanaya, "Coding theorems on correlated general sources," IEICE Trans. Fundamentals, vol.E78-A, no.9, pp.10631070, Sept. 1995

[9] N. Sato and H. Koga, "New results in optimistic source coding," IEICE Trans. Fundamentals, vol.E87-A, no.10, pp.2577-2580, Oct. 2004.

[10] D. Slepian and J. Wolf, "Noiseless coding of correlated information sources," IEEE Trans. Inf. Theory, vol.19, no.4, pp.471-480, 1973.

[11] S. Vembú, S. Verdú, and Y. Steinberg, "The source-channel transmission theorem revisited," IEEE Trans. Inf. Theory, vol.41, no.1, pp.44-54, 1995.

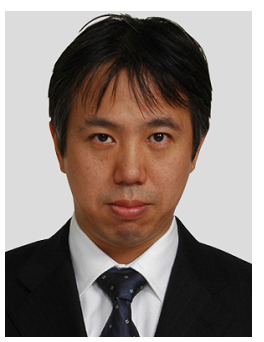

Hiroki Koga received B.E., M.E. and D.E degrees from University of Tokyo, in 1990, 1992 and 1995, respectively. From 1995 to 1999 , he was a Research Associate in Graduate school of Engineering, University of Tokyo. Since 1999, he has been with University of Tsukuba, where he is currently an Associate Professor of Graduate School of Systems and Information Engineering. His research interests are in Shannon theory and information security. Dr. Koga is a member of IEEE information theory society. 